\title{
Curcumin-incorporated Thiolated Chitosan/alginate Nanocarriers: Physicochemical Properties and Release Mechanism
}

\author{
C. PORNPITCHANARONG, NITJAWAN SAHATSAPAN, T. ROJANARATA, PRANEET OPANASOPIT, T. NGAWHIRUNPAT, \\ AND P. PATROJANASOPHON* \\ Pharmaceutical Development of Green Innovations Group (PDGIG), Faculty of Pharmacy, Silpakorn University, Nakhon \\ Pathom, 73000, Thailand
}

Pornpitchanarong et al.: Thiolated Chitosan/alginate Nanoparticles for Curcumin Delivery

\begin{abstract}
This study was aimed to develop thiolated chitosan/alginate nanoparticles for curcumin delivery. The nanoparticles were prepared from thiolated chitosan and sodium alginate using an ionic gelation technique. The concentrations of thiolated chitosan and alginate used to form the nanoparticles were optimal at 0.05 and $0.025 \% \mathrm{w} / \mathrm{v}$, respectively. Curcumin was incorporated into the nanoparticles by entrapment during nanoparticle preparation. The thiol content of the synthesized thiolated chitosan as well as the particle size, size distribution, surface charge, loading efficiency, loading capacity and drug release characteristics of the curcumin-loaded thiolated chitosan/alginate nanoparticles were investigated. Nano-sized particles ranging from 291-435 $\mathrm{nm}$ were obtained. The blank nanoparticles exhibited the smallest size and positive surface charge. Incorporation of curcumin into the nanoparticles resulted in a slightly bigger particle size and the surface charge became negative. After drug loading, the particle size was changed to $435 \mathrm{~nm}$ with the technique used. Sustained release of curcumin was observed from the nanoparticles. Kinetics of curcumin release fitted well with the zero-order model. Curcumin incorporated nanoparticles were successfully prepared with desirable physicochemical properties. Moreover, sustained release of curcumin from the nanoparticles with zero-order kinetic was obtained. Therefore, these nanoparticles may prove to be favourable carriers for curcumin delivery.
\end{abstract}

Key words: Curcumin, thiolated-chitosan, alginate, nanoparticles, colon cancer

Turmeric is widely used as a food spice and as an active component of traditional drug in Thai national list of essential medicine ${ }^{[1]}$. Turmeric rhizomes, which contain $\alpha \gamma$-turmerone, curcumin and its derivatives are reported to have various indications such as gastric astringents, antimicrobial, antiinflammatory and anticancer ${ }^{[2]}$. Many researchers investigated the anticarcinogenic activity of curcumin in animal models. It was proposed that curcumin could inhibit cancer cell initiation, which destructs gene mutation and also inhibits tumor promotion that accelerates cancer growth ${ }^{[3]}$. Moreover, inhibition of tumorigenesis in stomach, duodenum and colon has been proved from curcumin intake. In a study, mice were fed with curcumin prior to carcinogen treatment; it was found that mice receiving curcumin not only had less number of tumors, but also smaller tumor size $^{[4]}$. The anticarcinogenic mechanism of curcumin was reported to be inhibition of the colon carcinogenic

*Address for correspondence E-mail: patrojanasophon_p@su.ac.th January-February 2020 azoxymethane $\mathrm{e}^{[5]}$. Curcumin was not only found to be a potential anticarcinogenic agent, but it was also reported to enhance the efficacy of chemotherapeutic drugs.

Gastric cancer resistant to anticancer drugs was susceptible in vitro to anticancer agents when co-administered with curcumin; Additionally, chemotherapy drug dose reduction while given concurrent with curcumin could subsequently reduce side effects and increase drug tolerability in cancer patients ${ }^{[6]}$. Curcumin could also act as a P-glycoprotein inhibitor, which enhances anticancer

This is an open access article distributed under the terms of the Creative Commons Attribution-NonCommercial-ShareAlike 3.0 License, which allows others to remix, tweak, and build upon the work non-commercially, as long as the author is credited and the new creations are licensed under the identical terms

Accepted 30 November 2019

Revised 14 September 2019

Received 07 June 2019

Indian J Pharm Sci 2020;82(1):97-103 
effect of doxorubicin in drug-resistant gastric cancer cell line by inducing apoptotic cell death pathway ${ }^{[7]}$. Co-encapsulation of curcumin and doxorubicin in longcirculating liposomal formulation presented inhibitory mechanism of inflammatory and angiogenesis proteins involved in tumor growth ${ }^{[8]}$.

Curcumin is an orange-yellowish crystalline powder that is poorly soluble in water and ether, but very soluble in ethanol and glacial acetic acid ${ }^{[9]}$. Recently, various efforts have been made to deliver curcumin to the cancer cells. Zhang et al. investigated the use of curcumin-cyclodextrin complex to enhance water solubility and therapeutic efficacy of curcumin. In this study, curcumin-entrapped cyclodextrin induced cellular uptake and delayed release of curcumin in the lung cancer cell compared to free curcumin ${ }^{[10]}$. A study reported the development of curcuminloaded transferosome as a transdermal drug delivery system of curcumin for the treatment of breast cancer. Curcumin was also found to be a potential anticarcinogenic agent through skin penetration using a nanovesicle drug carrier ${ }^{[11]}$. Furthermore, curcumin was developed as a liposomal dry powder inhaler, which showed better therapeutic efficacy against primary lung cancer compared to free curcumin powder and chemotherapeutic agent, gemcitabine, both in vitro and in vivo ${ }^{[12]}$.

Nowadays, there have been numerous developments in drug delivery; however, polymer-based nano-systems seem to gain popularity. Polymeric nanoparticles (NPs) made of polymers with the size ranging from 10-1000 $\mathrm{nm}$, which is small enough to easily penetrate through intercellular gaps of tumors especially in tumours undergoing angiogenesis ${ }^{[13]}$. Resulting from increased blood vessel size, the intercellular gaps of cancer cells expand allowing passive targeting of a drug delivery system, which will enhance drug selectivity to the tumour as well as reduce adverse drug events ${ }^{[14]}$. Polymer-based NPs can be prepared from different types of polymers, for example, guar gum, dextran, chondroitin. Recently, mucoadhesive polymer drug delivery has gained interest due to its advantages over the conventional system. The first generation selfmucoadhesive polymers are chitosan (CS) and alginate (ALG); in addition to these, mucoadhesive polymers with modified functional groups for stronger binding to the mucous surface such as thiolated polymers or thiomers have been developed. Thiomers are polymers with thiol groups (-SH) incorporated into the polymer chain, rendering disulfide bond formation with the mucin glycoprotein aiming to enhance mucoadhesive property. Apart from the aforementioned information, thiomers act as penetration enhancer due to their capability to enlarge intercellular gaps to enable the polymer to penetrate ${ }^{[15]}$.

Herein, the development of thiolated chitosan (Cys-CS)/ALG NPs for curcumin delivery was reported, which could be used for colorectal cancer treatment. The mucoadhesive properties of Cys-CS could help increase the retention time of the NPs at the site of action providing the NPs to penetrate into the cancer cells by passive targeting. The particle size, size distribution, surface charge, loading efficiency, loading capacity and drug release characteristic of the curcumin-loaded Cys-CS/ALG NPs were investigated.

\section{MATERIALS AND METHODS}

Low molecular weight CS (75-85 \% deacetylated), sodium ALG from brown algae (viscosity of $2 \%$ solution at $25^{\circ} \sim 250 \mathrm{cps}$ ), L-cysteine hydrochloride, $N$-(3-dimethylaminopropyl)- $N$-ethylcarbodiimide hydrochloride (EDAC) and curcumin were obtained from Sigma Chemical Co. (St. Louis, MO, USA). All other reagents and solvents were of analytical grade and were used without further purification.

L-cysteine hydrochloride solution $(3.6 \mathrm{mM})$ was prepared in deionized water. Then, EDAC (3 mM) was added to the solution while the solution was stirring to activate the carboxylic group of cysteine. The reaction was continued for $20 \mathrm{~min}$. After that, $3.0 \mathrm{mM}$ CS solution in $1 \mathrm{w} \% \mathrm{HCl}$ was prepared before gently adding into the cysteine solution and the $\mathrm{pH}$ was adjusted to 4.0. The mixture was stirred for $6 \mathrm{~h}$ in the dark. Afterwards, the mixture was dialyzed against $1 \mathrm{mM} \mathrm{HCl}+1 \% \mathrm{w} / \mathrm{v} \mathrm{NaCl}$ solution on the first day and later against $1 \mathrm{mM} \mathrm{HCl}$ solution before being freeze dried (fig. 1).

The amount of thiol groups attached to the CS chain was determined by Ellman's assay. The synthesized Cys-CS $(10 \mathrm{mg})$ was soaked in $500 \mu \mathrm{l}$ phosphate buffer $(\mathrm{pH} 8.0)$ in a microcentrifuge tube. After that, $500 \mu 1$ of Ellman's reagent (5,5'-dithiobis(2-nitrobenzoic acid), $0.3 \mathrm{mg} / \mathrm{ml}$ ) was added. The mixture was shaken and incubated for 90 min protected from light. Cysteine hydrochloride solutions at different concentrations were prepared to generate a standard curve for thiol content analysis.

The NPs were prepared using ionic gelation method. CS was dissolved in $1 \% \mathrm{v} / \mathrm{v}$ acetic acid while ALG was dissolved in water to obtain the polymer solutions with 
different polymer concentrations. The $\mathrm{pH}$ of the $\mathrm{CS}$ and ALG solutions were adjusted to 5.0. Different ratios of CS:ALG (Table 1) were used to formulate the NPs. The ALG solution was added dropwise to the CS solution using a glass syringe fitted with a 25 gauge needle. The mixture was stirred for $1 \mathrm{~h}$ after ALG was completely added into the CS solution. The obtained mixture was freeze dried before the NPs were collected. The optimal ratio of CS:ALG was used to prepare Cys-CS NPs for further investigations.

The particle size and zeta potential of the prepared NPs were evaluated at $25^{\circ}$ using a Zetasizer Nano ZS (Malvern Instruments, Malvern, UK). Sample solutions were diluted to $1: 10$ with deionized water and probe sonicated to prevent particle aggregation before being filled into $1-\mathrm{cm}^{3}$ zeta cell. These measurements were made in triplicate.

Cys-CS solution and ALG solution of the selected ratio were prepared as stated earlier. Curcumin solution $(1 \mathrm{mg} / \mathrm{ml})$ in ethanol was slowly added to the ALG solution using a micropipette before dropping the mixture into the Cys-CS solution according to the preparation method mentioned above. To quantify the curcumin loaded in the NPs, $1 \mathrm{ml}$ of ethanol was added to the NPs and the mixture was shaken overnight to extract curcumin from the NPs. Then the mixture was centrifuged for $30 \mathrm{~min}$ and the supernatant was collected. The extent of curcumin entrapped in the NPs was quantified using a UV/Vis spectrophotometer at $419 \mathrm{~nm}$. The loading efficiency and loading capacity were computed according the Eqns. 1 and 2, respectively. Loading efficiency $(\%)=$ the amount of curcumin in the NPs $\times 100$ /initial amount of curcumin used. Loading capacity $=$ the amount of curcumin in the NPs/the weight of the polymer used.

The chemical structure of the Cys-CS, blank NPs, and curcumin-loaded NPs was characterized by Fouriertransformed infrared spectroscopy (FTIR, Perkin Elmer spectrum 100) between $600-4000 \mathrm{~cm}^{-1}$. The samples were pressed with potassium bromide to form clear disc and locked onto a holder prior to the observation. Proton nuclear magnetic resonance spectroscopy ( ${ }^{1} \mathrm{H}-\mathrm{NMR}$, $300 \mathrm{MHz}$ Avance III HD, Bruker) at $298 \mathrm{~K}$ was also performed to elucidate the structure of the particles and components. Each sample was completely dissolved in deuterium oxide $\left(\mathrm{D}_{2} \mathrm{O}\right)$ for the investigation and all data were recorded as chemical shift in parts per million (ppm; $\mathrm{D}_{2} \mathrm{O}$ chemical shift $=4.80 \mathrm{ppm}$ ). The thermal properties and molecular dispersion of curcumin, blank NPs, and curcumin-loaded NPs were examined using differential scanning calorimetry (DSC, Perkin Elmer DSC 8000, Waltham, MA, USA). Accurately weighed samples in a solid pan were placed in the instrument with temperature programmed from $20-250^{\circ}$, rate $10 \% \mathrm{~min}^{[16,17]}$. Scanning electron microscope (SEM, Tescan Mira 3, Czech Republic) was performed to analyse the morphological characteristics of the NPs. Blank NPs were covered with thin gold layer after fixing on a metal stub using two-sided tape prior to the observation.
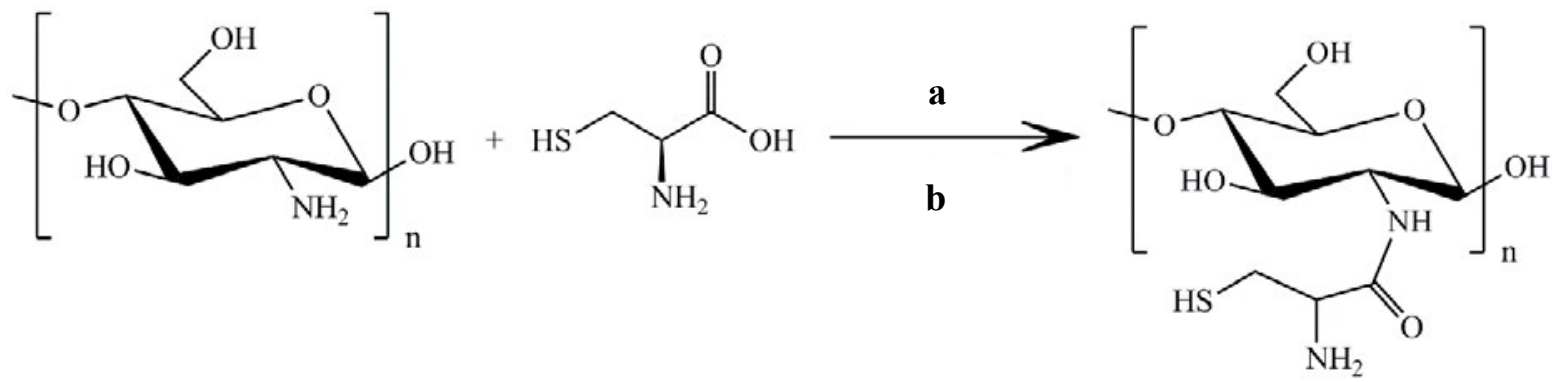

Fig. 1: The synthetic route of Cys-CS conjugates

(a) $25 \%$ h, (b) EDAC/NHS/ $/ \mathrm{H}_{2} \mathrm{O}$

TABLE 1: SIZE, SIZE DISTRIBUTION AND ZETA POTENTIAL OF THE NANOPARTICLES

\begin{tabular}{|c|c|c|c|c|c|c|}
\hline \multirow{2}{*}{ Formulations } & \multicolumn{3}{|c|}{ Polymer concentration (\% w/v) } & \multirow{2}{*}{ Particle size $(\mathrm{nm})$} & \multirow{2}{*}{ PDI } & \multirow{2}{*}{ Zeta potential $(\mathrm{mV})$} \\
\hline & CS & Cys-CS & ALG & & & \\
\hline $\mathrm{F} 1$ & 0.025 & - & 0.025 & $124.7 \pm 0.4$ & $0.19 \pm 0.01$ & $+30.30 \pm 2.62$ \\
\hline $\mathrm{F} 2$ & 0.05 & - & 0.025 & $110.8 \pm 0.4$ & $0.25 \pm 0.01$ & $+35.50 \pm 3.04$ \\
\hline $\mathrm{F} 3$ & 0.05 & - & 0.05 & $124.1 \pm 3.0$ & $0.33 \pm 0.01$ & $+37.63 \pm 2.06$ \\
\hline $\mathrm{F} 4$ & 0.025 & - & 0.05 & $242.3 \pm 21.3$ & $0.40 \pm 0.05$ & $+25.70 \pm 2.72$ \\
\hline F5 & - & 0.05 & 0.025 & $291.9 \pm 5.0$ & $0.37 \pm 0.01$ & $+18.70 \pm 1.08$ \\
\hline CUR-loaded F5 & - & 0.05 & 0.025 & $435.2 \pm 129.7$ & $0.41 \pm 0.13$ & $-1.07 \pm 0.78$ \\
\hline
\end{tabular}


Freeze-dried NPs containing curcumin were accurately weighed $(20 \mathrm{mg})$ and dispersed in $1 \mathrm{ml}$ of release medium pH 7.4 (phosphate buffered saline (PBS) with $1 \%$ Tween 20) contained in a dialysis bag. Then, the dialysis bag was submerged in a glass bottle containing $13 \mathrm{ml}$ of the release medium and being shaken at 200 $\mathrm{rpm}$ in a shaker incubator at $37^{\circ}$. At different time intervals of 5, 15, $30 \mathrm{~min}, 1,2,4,8 \mathrm{~h}, 1,2,3,4,5,6,7 \mathrm{~d}$, an aliquot $(1.5 \mathrm{ml})$ was removed, and the equal volume of fresh medium was added to maintain the initial volume. The quantities of curcumin released to the release medium at different time points were measured using a UV/Vis spectrophotometer operated at 419 $\mathrm{nm}$. The experiments were carried out in triplicate and the release kinetics of curcumin from the NPs were analysed using the zero-order model, the first-order model and the Higuchi model.

\section{RESULTS AND DISCUSSION}

The structure of Cys-CS conjugate synthesized by coupling $-\mathrm{COOH}$ group of cysteine $\mathrm{HCl}$ with the $-\mathrm{NH}_{2}$ groups of $\mathrm{CS}$ was confirmed using ${ }^{1} \mathrm{H}-\mathrm{NMR}$ and FTIR as shown in figs. 2 and 3, respectively. From the ${ }^{1} \mathrm{H}-\mathrm{NMR}$ spectra, it was confirmed that cysteine was successfully grafted on the CS chain, which can be observed from the proton of the amino acid $\alpha$-carbon and carbon of the side chain presented at 2.83 and $3.60 \mathrm{ppm}$, respectively. The FTIR spectra of CS and

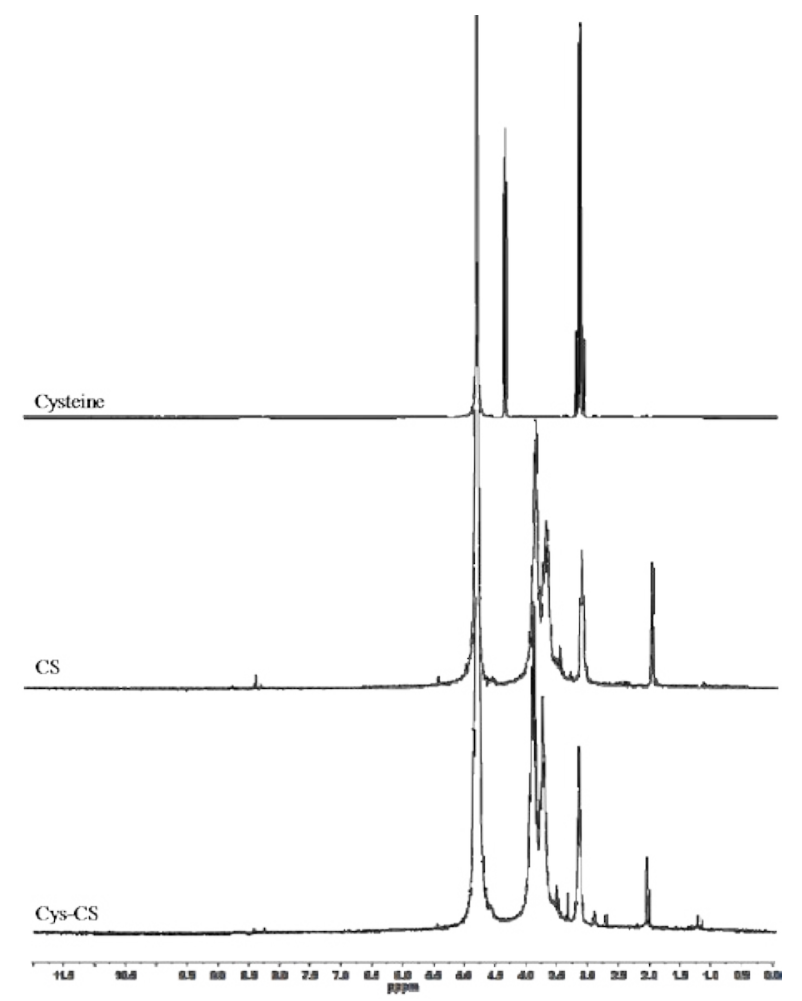

Fig. 2: ${ }^{1} \mathrm{H}-\mathrm{NMR}$ spectra of cysteine, CS, and Cys-CS

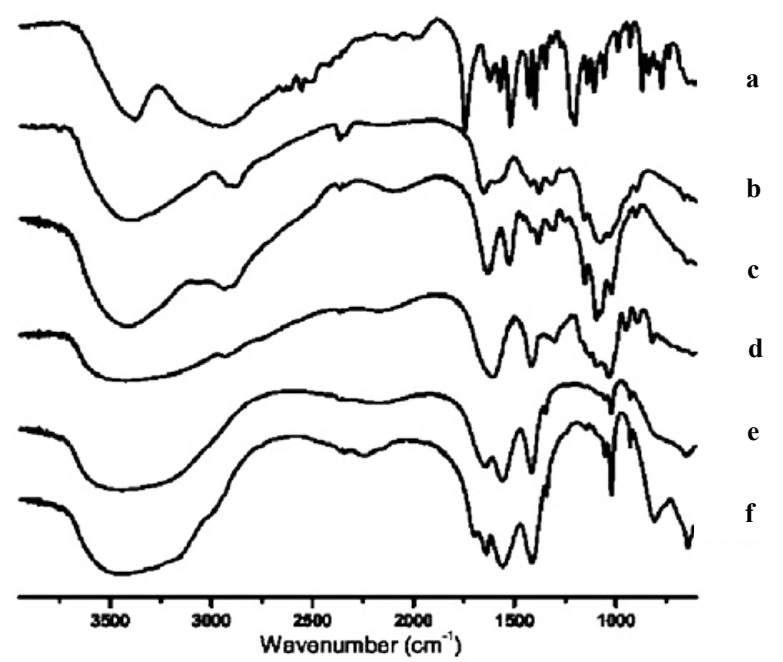

Fig. 3: FT-IR spectra

(a) Cysteine, (b) CS, (c) Cys-CS, (d) ALG, (e) blank NPs, and (f) curcumin-loaded NPs

Cys-CS exhibited the band of amide carbonyl group at $1640 \mathrm{~cm}^{-1}$. The peaks at $3389 \mathrm{~cm}^{-1}$ is due to $\mathrm{O}-\mathrm{H}$ stretching. The bands at 2890 and $1075-1034 \mathrm{~cm}^{-1}$ correspond to $\mathrm{C}-\mathrm{H}$ and $\mathrm{C}-\mathrm{O}$ stretching of pyranose ring. Although the peak of thiol group in the Cys-CS could not be seen due to the small amount of cysteine attached to the CS backbone, the thiol content was quantified using Ellman's assay to confirm successful synthesis. The thiol content of the synthesized Cys-CS was found to be $253 \mu \mathrm{mol} / \mathrm{g}$.

The NPs were formed through ionic gelation technique using negatively charged carboxylate functional group to interact with positively charged CS amino group at various ratios of ALG and CS. The particle size, size distribution and zeta potential of the NPs are presented in Table 1. All NPs obtained were nano-sized with positive surface charge from the amino group of CS. Considering NPs sizes, once greater ratio of CS was used to form the NPs (CS:ALG, 2:1), the blank NPs exhibited smallest particles with positive charge. Therefore, this ratio was selected to prepare the Cys-CS/ALG NPs for further investigations. The CysCS NPs were somewhat larger than the CS/ALG NPs at the same ratio; however, the size remained within the preferable nano-range. This may be due to the lower quantity of positively charged amines on the CS backbone due to the replacement by cysteine. This is confirmed by the lessen positive zeta potential of the Cys-Cs/ALG NPs. The FTIR spectrum of the blank Cys-CS NPs (fig. 3) corresponded to the Cys-CS showing $\mathrm{C}=\mathrm{O}$ amide bond characteristic at $1640 \mathrm{~cm}^{-1}$ together with ALG characteristic of carboxylic $\mathrm{O}-\mathrm{H}$ bending at $1420 \mathrm{~cm}^{-1}$. The successful loading of curcumin into 


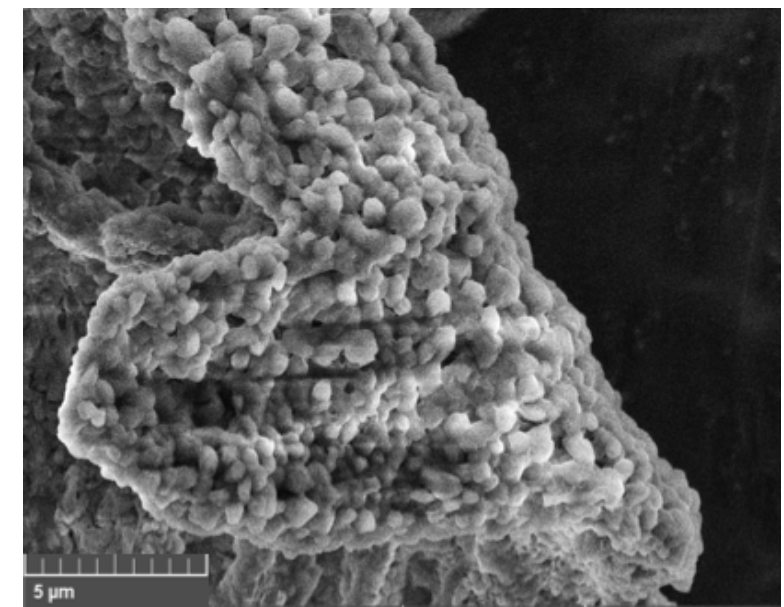

Fig. 4: SEM micrograph of Cys-CS/ALG NPs

the NPs was asserted by the presence of $\mathrm{C}=\mathrm{O}$ aliphatic ketone at $1705 \mathrm{~cm}^{-1}$. The SEM image as shown in fig. 4 presented the morphology of the Cys-CS/ALG NPs. It can be observed that the particles were in spherical shape, however, the hydrophilic nature of the polymers caused the particles to aggregate after the lyophilization process. However, the particles segregated after redispersion in water as proven by the narrow PDI of the particle. The DSC thermogram of curcumin, the blank NPs, and the curcumin-loaded NPs are displayed in fig. 5. Curcumin showed melting temperature at $176^{\circ}$. The blank NPs showed no dominant thermal characteristics apart from the melting of polymer components, whereas, the curcumin-loaded NPs presented the melting temperature at lower temperature, which may be associated with the dispersion of curcumin in the NPs. The reason behind this phenomenon is due to the solid dispersion technique of increasing the solubility of an active compound after curcumin was mixed with ALG solution and transformed to amorphous state. This favours the solubility of the poorly soluble curcumin and may improve the efficacy of the formulation. Incorporation of curcumin into the NPs led to the increase in the particle size. This could be because curcumin may uniformly disperse in the NPs. Despite the increment, the obtained particle size seems to be optimal according to literatures stating the enhance permeability and retention effect of the tumour may also accumulate the NPs within the tumor ${ }^{[18,19]}$. The surface charge of the NPs became slightly negative after curcumin was loaded due to the negative charge of curcumin. The sparingly anionic surface charge would protect the formulation from membrane adsorption and cellular uptake and these are less likely to induce nonphagocytotic cell disruption. Moreover, the negative surface charge leads to prolonged circulation period and enhance accumulation of the NPs in the tumor cells $^{[20,21]}$.

Loading efficiency and loading capacity of the curcuminloaded Cys-CS/ALG NPs was found to be $2.80 \pm 0.18 \%$ and $5.61 \pm 0.41 \mu \mathrm{g} / \mathrm{mg}$, respectively. Drug entrapment may be resulted from the difference between the charge of the NPs (positive charge) and curcumin (negative charge) enabling a network entrapping curcumin. Factors having effect on drug loading efficacy and capacity include drug solubility, molecular weight and importantly the interaction between drug and polymer ${ }^{[22]}$. Curcumin is poorly soluble in water, which might be the cause of limited drug loading found. Although highest loading efficiency possible might be desired in drug delivery design, but in vivo pharmacokinetic study revealed that optimal drug loading may be preferential for less drug accumulation in the organs ${ }^{[19,23]}$. This study demonstrated that curcumin can be loaded onto the NPs through the entrapment method and further in vivo study would be necessary to elucidate the suitability of drug dosing.

The release of curcumin form the NPs was performed in PBS pH 7.4 containing $1 \%$ Tween 20, and the results are displayed in fig. 6. As it can be seen from the results, curcumin released from the NPs in a sustain manner. Just above $90 \%$ of the curcumin contained in the NPs release to the dissolution medium at a prolong period of $1 \mathrm{w}$.

Once the drug release profile was obtained, it was fit to different release kinetic models and the findings are presented in fig. 7. The release of curcumin from curcumin-loaded NPs prepared by entrapment method best fit with the zero order model with $\mathrm{R}^{2}$ of 0.983 , which describes the drug release from a drug delivery system containing non-swellable system with hydrophobic $\operatorname{drug}^{[24]}$.

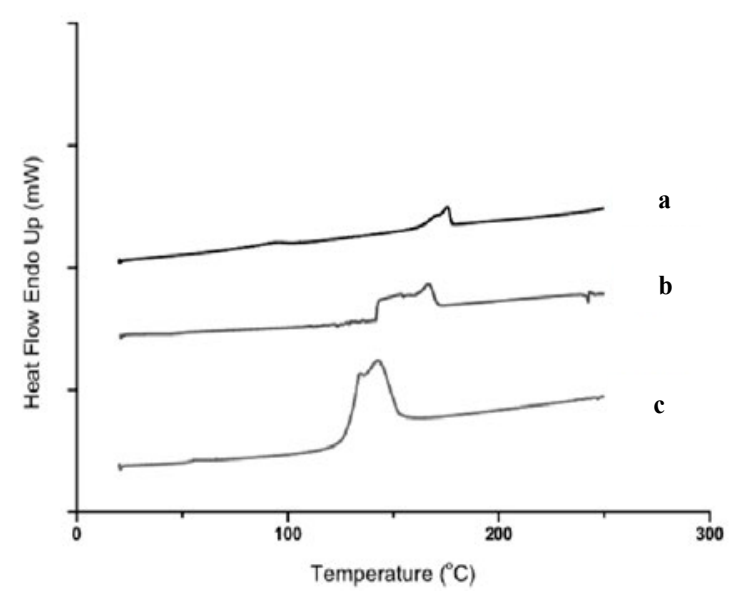

Fig. 5: DSC thermograms of (a) curcumin, (b) blank NPs, and (c) curcumin-loaded NPs 


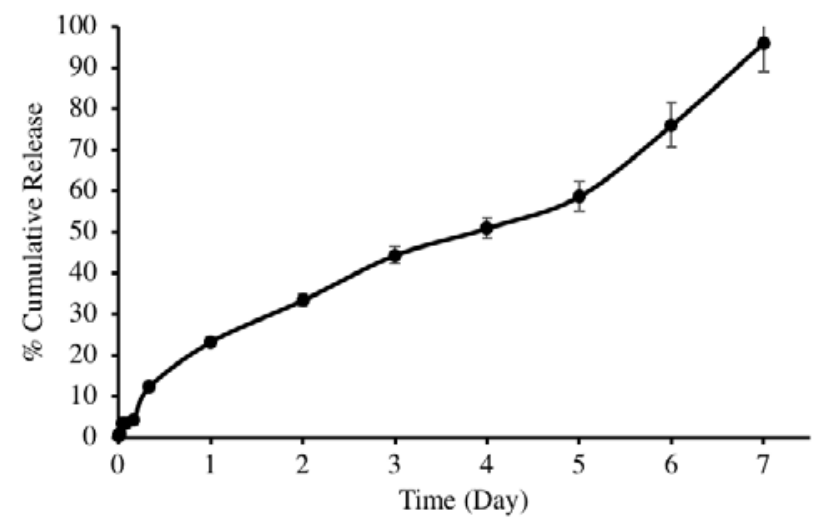

Fig. 6: Release characteristic of curcumin from the Cys-CS/ ALG NPs prepared using entrapment method
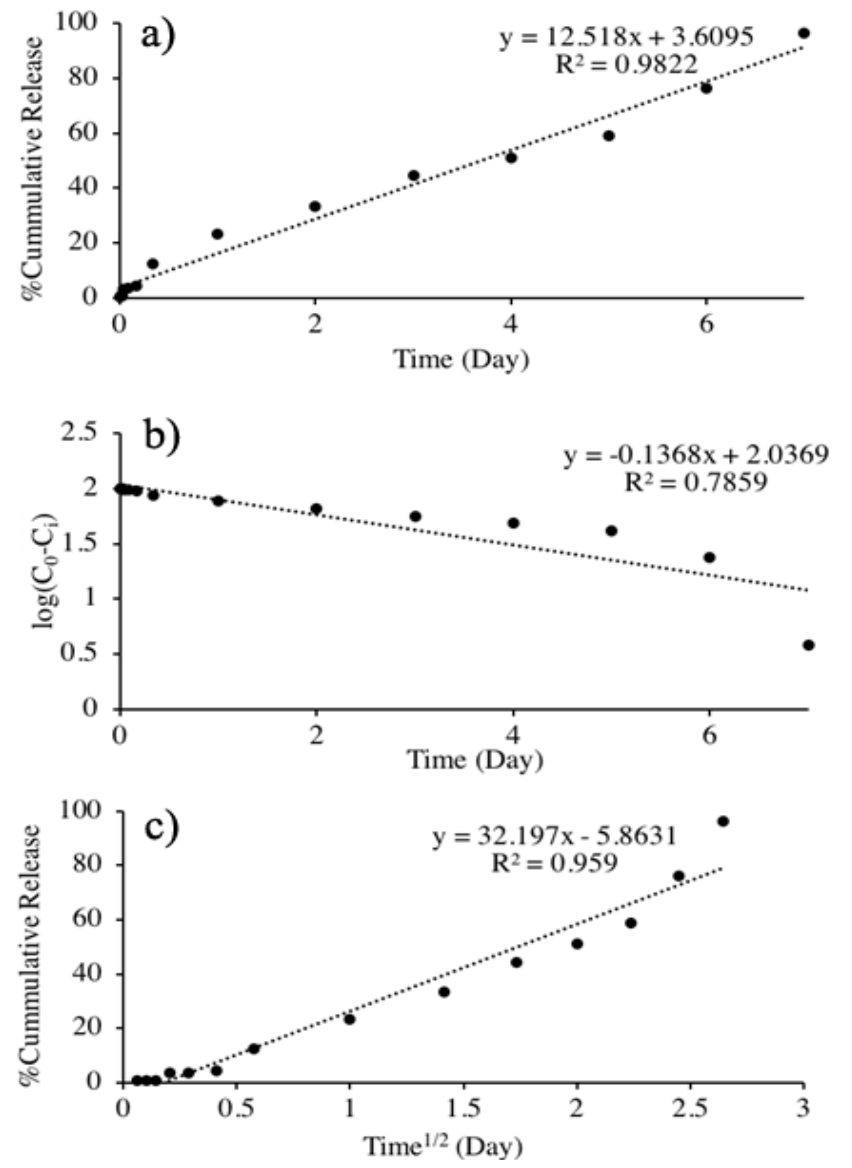

Fig. 7: Kinetic models of curcumin release from the NPs with drug loading by entrapment technique (a) zero order model, (b) first order model, and (c) Higuchi model

Long period, up to one week, of the drug release is desirable for cancer treatment delivery system because it would allow additional effectiveness, reduce side effects and improve patient compliance. Loading curcumin by entrapment method offered sustainable and favourable drug release, which $90.11 \%$ of the curcumin loaded was released to the release medium in $7 \mathrm{~d}$. Moreover, drug release kinetics revealed zero order. This may be a consequence of the crosslink
Cys-CS/ALG resulting in the constant release of curcumin, which evenly dispersed in the NPs. Apart from that, drug solubility can be a factor affecting the release of curcumin as it is a hydrophobic compound, solubility may become the release rate-limiting step ${ }^{[22]}$. Near to the ideal kinetic model, curcumin release would minimize initial burst and prolong therapeutic efficacy ${ }^{[25]}$.

Curcumin incorporated NPs was successfully prepared by ionic gelation method. The nanosized particles were obtained with slightly negative surface charge after curcumin was incorporated. Incorporation of curcumin by entrapment technique led to the increased particle size and desirable release of curcumin in acceptable range, which is best fit with the zero-order kinetic model. Therefore, the curcumin-loaded NPs prepared by entrapment method might provide a promising strategy to develop a control release drug delivery system for colorectal cancer treatment. However, the anticancer activity in vitro and in vivo should be further investigated.

\section{Conflict of interest:}

The authors report no declarations of interest.

\section{Acknowledgments:}

The authors would like to acknowledge the Commission of Higher Education (Thailand) and the Thailand Research Funds through the Golden Jubilee Ph.D. Program (Grant No.PHD/0021/2560), the Office of the Higher Education Commission and the Thailand Research Funds (Grant no. MRG 6180182), and the Thailand Research Funds through the Research Team Promotion Grant (RTA6180003). Special thanks to Ms. Nattaya Kasirak, Ms. Patteera Tungtong, Ms. Natnaree Phuklewkla, and Mr. Phattharapol Emrat for research assistance.

\section{REFERENCES}

1. Department of drug Thai food and drug administration Thailand Ministry of Health. NLEM: Herbal Medicines 2013. [cited 2017 Sep 27]. Available from: http://kpo.moph.go.th/ webkpo/tool/Thaimed2555.pdf.

2. Curcumin, Med Plant Faculty of Pharmacy Mahidol University. [cited 2017 Sep 27]. Available from: http://www.medplant. mahidol.ac.th/pubhealth/curcuma.html.

3. Hamzehzadeh L, Atkin SL, Majeed M, Butler AE, Sahebkar A. The versatile role of curcumin in cancer prevention and treatment: A focus on PI3K/AKT pathway. J Cell Physiol 2018;233(10):6530-7.

4. Troselj KG, Kujundzic RN. Curcumin in Combined Cancer Therapy. Curr Pharm Des 2014;20(42):6682-96.

5. Wong KE, Ngai SC, Chan KG, Lee LH, Goh BH, Chuah LH. 
Curcumin Nano formulations for Colorectal Cancer: A Review. Front Pharmacol 2019;10:152.

6. Ebrahimifar M, Hasanzadegan-Roudsari M, Kazemi SM, Ebrahimi-Shahmabadi H, Kanaani L, Alavi SA, et al. Enhancing Effects of Curcumin on Cytotoxicity of Paclitaxel, Methotrexate and Vincristine in Gastric Cancer Cells. Asian Pac J Cancer Prev 2017;18(1):65-8.

7. Huang R, Yu H, Hu F, Tian S. Strategy to enhance efficacy of doxorubicin by curcumin as a potent Pgp inhibitor in gastric cancer. Biomed Res 2017;28(3):1231-6.

8. Sesarman A, Tefas L, Sylvester B, Licarete E, Rauca V, Luput $\mathrm{L}$, et al. Anti-angiogenic and anti-inflammatory effects of long-circulating liposomes co-encapsulating curcumin and doxorubicin on C26 murine colon cancer cells. Pharmacol Rep 2018;70(2):331-9.

9. O’Neil MJ. The Merck Index - An Encyclopedia of Chemicals, Drugs, and Biologicals. Cambridge, UK: Royal Society of Chemistry; 2013.

10. Zhang L, Man S, Qiu H, Liu Z, Zhang M, Ma L, et al. Curcumin-cyclodextrin complexes enhanced the anti-cancer effects of curcumin. Environ Toxicol Pharmacol 2016;48:31-8.

11. Abdel-Hafez SM, Hathout RM, Sammour OA. Curcuminloaded ultradeformable nanovesicles as a potential delivery system for breast cancer therapy. Colloids Surf B Biointerfaces. 2018;167:63-72.

12. Zhang T, Chen Y, Ge Y, Hu Y, Li M, Jin Y. Inhalation treatment of primary lung cancer using liposomal curcumin dry powder inhalers. Acta Pharm Sin B 2018;8(3):440-8.

13. Golombek SK, May JN, Theek B, Appold L, Drude N, Kiessling $\mathrm{F}$, et al. Tumor targeting via EPR: Strategies to enhance patient responses. Adv Drug Deliv Rev 2018;130:17-38.

14. Nunthanid J. Targeted drug delivery. Thai Bull Pharm Sci 2002;8:19-28

15. Tonglairoum P, Opanasopit P. Thiomers: a new generation mucoadhesive polymer for transmucosal drug delivery system. Thai Bull Pharm Sci 2014;8:19-28.

16. Rachmawati H, Yanda YL, Rahma A, Mase N. CurcuminLoaded PLA Nanoparticles: Formulation and Physical Evaluation. Sci Pharm 2016;84(1):191-202.

17. Jahagirdar PS, Gupta PK, Kulkarni SP, Devarajan PV. Polymeric curcumin nanoparticles by a facile in situ method for macrophage targeted delivery. Bioeng Transl Med 2019;4(1):141-51.

18. Medina-Alarcon KP, Voltan AR, Fonseca-Santos B, Moro IJ, de Oliveira Souza F, Chorilli M, et al. Highlights in nanocarriers for the treatment against cervical cancer. Mater Sci Eng C Mater Biol Appl 2017;80:748-59.

19. Pornpitchanarong $C$, Rojanarata $T$, Opanasopit $P$, Ngawhirunpat T, Patrojanasophon P. Synthesis of novel N-vinylpyrrolidone/ acrylic acid nanoparticles as drug delivery carriers of cisplatin to cancer cells. Colloids and Surfaces B: Biointerfaces 2020;185:110566.

20. Fröhlich E. The role of surface charge in cellular uptake and cytotoxicity of medical nanoparticles. Int $\mathrm{J}$ Nanomedicine 2012;7:5577-91.

21. Blanco E, Shen H, Ferrari M. Principles of nanoparticle design for overcoming biological barriers to drug delivery. Nat Biotechnol 2015;33(9):941-51.

22. Singh R, Lillard JW Jr. Nanoparticle-based targeted drug delivery. Exp Mol Pathol 2009;86(3):215-23.

23. Petschauer JS, Madden AJ, Kirschbrown WP, Song G, Zamboni WC. The effects of nanoparticle drug loading on the pharmacokinetics of anticancer agents. Nanomedicine 2015;10(3):447-63.

24. Bruschi ML. Strategies to Modify the Drug Release from Pharmaceutical Systems. 1st ed. Sawston, United Kingdom, Cambridge, United Kingdom; Woodhead Publishing; 2015. p. 63-86.

25. Zhao YN, Xu X, Wen N, Song R, Meng Q, Guan Y, et al. A Drug Carrier for Sustained Zero-Order Release of Peptide Therapeutics. Sci Rep 2017;7(1):5524. 